\title{
Multiscale Causal Connectivity Analysis by Canonical Correlation: Theory and Application to Epileptic Brain
}

\author{
Guo Rong Wu, Fuyong Chen, Dezhi Kang, Xiangyang Zhang, Daniele Marinazzo*, and Huafu Chen*
}

\begin{abstract}
Multivariate Granger causality is a well-established approach for inferring information flow in complex systems, and it is being increasingly applied to map brain connectivity. Traditional Granger causality is based on vector autoregressive (AR) or mixed autoregressive moving average (ARMA) model, which are potentially affected by errors in parameter estimation and may be contaminated by zero-lag correlation, notably when modeling neuroimaging data. To overcome this issue, we present here an extended canonical correlation approach to measure multivariate Granger causal interactions among time series. The procedure includes a reduced rank step for calculating canonical correlation analysis (CCA), and extends the definition of causality including instantaneous effects, thus avoiding the potential estimation problems of AR (or ARMA) models. We tested this approach on simulated data and confirmed its practical utility by exploring local network connectivity at different scales in the epileptic brain analyzing scalp and depth-EEG data during an interictal period.
\end{abstract}

Index Terms-Canonical correlation analysis, depth-EEG, multivariate Granger causality.

\section{INTRODUCTION}

A FULL understanding of the brain network requires not only an understanding of correlated relationships between the separated neuronal populations but also an understanding of changes in inter-regional interactions. Functional connectivity investigates the connections between brain regions using mea-

Manuscript received April 2, 2011; revised June 8, 2011; accepted July 6, 2011. Date of publication July 22, 2011; date of current version October 19 2011. This work is supported in part by the Natural Science Foundation of China (61035006, 30900326 and 90820006), and by Program for Chang Jiang Scholars and Innovative Research Team in University. Asterisk indicates corresponding author.

G. R. Wu is with the Key Laboratory for NeuroInformation of Ministry of Education, School of Life Science and Technology, University of Electronic Science and Technology of China, Chengdu 610054, China (e-mail: wugr@uestc.edu.cn).

F. Chen and D. Kang are with the Department of Neurosurgery, the First Affiliated Hospital of Fujian Medical University, Fuzhou 350005, China (e-mail: dr_fychen@163.com; Kdz99988@sina.com).

$\mathrm{X}$. Zhang is with the Department of Psychiatry and Behavioral Sciences, Baylor College of Medicine, Houston, TX, USA (e-mail: xyzhang@bcm.edu).

${ }^{*}$ D. Marinazzo is with the Department of Data Analysis, Faculty of Psychology and Educational Sciences, Ghent University, 1 Henri Dunantlaan B 9000 Gent, Belgium (e-mail: daniele.marinazzo@ugent.be).

${ }^{*} \mathrm{H}$. Chen is with the Key Laboratory for NeuroInformation of Ministry of Education, School of Life Science and Technology, University of Electronic Science and Technology of China, Chengdu 610054, China (e-mail: chenhf@uestc.edu.cn).

Color versions of one or more of the figures in this paper are available online at http://ieeexplore.iee.org.

Digital Object Identifier 10.1109/TBME.2011.2162669 sures of statistical dependencies such as correlation or partial correlation or mutual information. These measures are in general nondirectional. Effective connectivity measures on the other hand rely on model estimation, are in general directed, and have become increasingly relevant in neuroimaging [1]-[3]. In recent years, many methodological advances have been applied to this type of connectivity analysis, including structural equation modeling [4], dynamic causal models [5], and Granger causality (GC) [6]-[10]. The first two methods rely on anatomically and physiologically based models, and their successful application depends on the exact obeissance to these constraints [11]. On the other hand, Granger causality is a data-driven method, first proposed and formalized by Granger [12]-[14]. GC relies on temporal precedence (the cause occurs before the effect) and is based on the notion of the improved predictability of one signal when information about another one is added to the model [12], [13]. GC is broadly used in neuroscience [7], [15]. In spite of the successes of Granger's method, some key theoretical problems remain unsolved. Previous approaches to detect Granger causality, e.g., dynamic vector autoregressive (VAR), sparse VAR, and nonlinear VAR have been suggested to extend the standard framework of VAR model based Granger causality [16]. More recently, multivariate Granger causality has been proved to be an effective and powerful tool to detect information flow among different components in complex systems [17]-[21], such as multivariate VAR, kernel Granger causality [18], [22], canonical correlation [19], [20], and kernel canonical correlation [23]. As the choice of an appropriate order of VAR model is not trivial nor univocal, and may affect the outcomes of the test [24], the aforementioned VAR-based Granger causality tests could not be able to balance variance accounted for against model complexity. Furthermore, it has been proposed that the traditional multivariate Granger causality may suffer the "leakage" of zero-lag correlation into the causal domain [25], [26]. It has been suggested that cross-correlation and canonical correlation could overcome this potential estimation problem [20], [27]. However, the cross-correlation method is a univariate approach [27], whereas the approach based on canonical correlation may be limited to Gaussian processes [20]. Partial canonical correlation [17], [21] could also be unsuccessful in excluding instantaneous effects in traditional Granger causality analysis. In order to overcome these issues, here we present an extended canonical correlation approach for multivariate Granger causality analysis. The approach is first tested on simulated data. Then we apply the proposed method in scalpEEG and depth-EEG data from an epileptic patient, and explore 
the pattern of effective connectivity at different scales during interictal period.

\section{METHOD}

\section{A. CCA Bivariate Granger Causality Analysis}

Let $\left\{\Omega_{t}\right\}$ be a set of information from an entire ensemble of concurrent stochastic processes, and $\left\{\Im_{t}\right\}$ be a subset of $\left\{\Omega_{t}\right\}$ including at least the stochastic processes $\left\{X_{t}, Y_{t}\right\}$. The subscript indicates the time. If the model which uses information from both $X_{t}$ and $Y_{t}$ yields a better prediction of the future values of $X$ than the model which uses only information about $X_{t}$, then this means that the series $Y$ contains some information about $X$ which is not available elsewhere, and thus $Y$ is said to cause $X$ [28]. In practice, we cannot deal with the complete set $\Omega_{t}$, so $\Im_{t}$ is used in substitution. In the application, regression techniques and cross-correlation are the main tool to identify Granger causality flow, and the standard test of GC is based on VAR model [13], [29].

Let us assume that the variables $X$ and $Y$ are wide-sense stationary multivariate stochastic processes. Consider the pair of multivariate autoregressive models [2], [13]-[15]

$$
\begin{aligned}
& X_{t}=\sum_{i=1}^{\infty} a_{1 i} X_{t-i}+\varepsilon_{t} . \\
& X_{t}=\sum_{i=1}^{\infty} a_{2 i} X_{t-i}+\sum_{j=1}^{\infty} b_{2 i} Y_{t-j}+\varepsilon_{t}^{\prime} .
\end{aligned}
$$

Then $Y_{t}$ Granger causes $X_{t}$, if $\left|\Sigma\left(\varepsilon_{t}\right)\right| \succ\left|\Sigma\left(\varepsilon_{t}^{\prime}\right)\right|$, that is, if the generalized variance of the error from model (2) is statistically smaller than the generalized variance of the error from model (1) [30]. The standard test of GC is defined by the logarithm of residual ratio between the regression models (1) and (2) [13], [30]. Theoretical autoregressive processes have infinite model order, but we should estimate a number of lagged observations from empirical time series data. Akaike information criterion [31], Bayesian information criterion [32], Hannan-Quinn criterion [33], cross validation [34], [35], and other order selection criteria have been developed for the selection of order. Standard Granger causality only considers the lagged effects among the time series, and neglects the instantaneous (not lagged) effects, which implies that any zero-lag correlation among the time series is translated into a correlation among the model residuals [13], [25], [26], [29]. Geweke introduced a transformation to the noise covariance to account for the instantaneous correlation, which leads to a decomposition where the total interdependence between $x$ and $y$ can be decomposed into " $x$ drives $y$ ", " $y$ drives $x$ ", and instantaneous causality [36], [37]. To address this distinction in the current framework, here we consider the model including the instantaneous effects, and reformulate (2) as follows [25]:

$$
X_{t}=\sum_{i=1}^{\infty} a_{2 i} X_{t-i}+\sum_{j=0}^{\infty} b_{2 i} Y_{t-j}+\varepsilon_{t}^{\prime \prime}
$$

then,

$$
\begin{aligned}
\tilde{X}_{t} & =X_{t}-b_{20} Y_{t}=\sum_{i=1}^{\infty} a_{2 i} X_{t-i}+\sum_{j=1}^{\infty} b_{2 i} Y_{t-j}+\varepsilon_{t}^{\prime \prime} \\
& =\left[X_{t-\text { past }}, Y_{t-\text { past }}\right]\left[\begin{array}{l}
A_{1} \\
B_{1}
\end{array}\right]+\varepsilon_{t}^{\prime \prime} .
\end{aligned}
$$

In the generalized multiple linear regression equation (3), $X_{t-\text { past }}\left(X_{t-\text { past }}=\left[X_{t-1}, \ldots, X_{t-n}, \ldots\right]\right)$, and $Y_{t-p a s t}$ $\left(Y_{t-\text { past }}=\left[Y_{t-1}, \ldots, Y_{t-n}, \ldots\right]\right)$ are the lagged distributions of $X_{t}$ and $Y_{t}$, respectively. If $B_{1}$ is statistically significant, $Y_{t}$ Granger causes $X_{t}$; furthermore, there is a significant correlation between $\tilde{X}_{t}$ and $Y_{t-\text { past }}$, partialing out the effect of $X_{t-\text { past }}$.

Canonical correlation analysis (CCA) is a method of correlating linear relationships between two multidimensional variables in feature space [38], [39]. In a sense, multiple linear regression is a special case of canonical correlation analysis [40]. The relationship between canonical correlation and Granger causality in the case of Gaussian processes has been established in the framework of information theory [19], [20]. Canonical correlation based Granger causality has also been used to study gene expression and functional magnetic resonance imaging (fMRI) signals [17], [21]. Due to the small sample size and high dimensionality of the dataset, those studies were limited to the information of one time point lag distribution [21]. Lagged values of the corresponding variables are taken into account in order to diminish the risk caused by spurious regressions [41]. Information computed with an embedding dimension of only one may be insufficient to avoid spurious correlations in applications where the instantaneous effect is strongly present. To overcome these issues in the following, we present an extended canonical correlation approach for multivariate Granger causality analysis.

Consider the random vector $C=\left(\hat{X}_{t}, \hat{Y}_{t-\text { past }}\right)$ on $\mathrm{H} \subseteq \Re^{q}$, with the covariance matrix $\Sigma=\left(\Sigma_{i j}\right)$ (where $\Sigma_{i j}(i, j=1,2)$ denotes the partitioned matrix). We can assume without loss of generality that each column contains values with zero-mean and unit variance. Linear CCA seeks a pair of basis vectors $\alpha \in \Re^{q_{1}}$ and $\beta \in \Re^{q_{2}}$ for the $\hat{X}_{t}$ and $\hat{Y}_{t-\text { past }}$, such that the correlations between $\hat{X}_{t} \alpha$ and $\hat{Y}_{t-\text { past }} \beta$ are mutually maximized, namely, it solves the following optimization problem:

$$
\begin{aligned}
& \rho:=\max _{[\alpha ; \beta] \in \Re^{q_{1}+q_{2}}} \alpha^{T} \Sigma_{12} \beta \\
& \quad \text { subject to } \alpha^{T} \Sigma_{11} \alpha=1 \text { and } \beta^{T} \Sigma_{22} \beta=1
\end{aligned}
$$

which could be simply solved by linear algebra techniques [38]. The canonical correlation coefficient is given by $\rho=$ $\left(\beta^{T} \beta\right)^{-1} \beta^{T} B_{1} \alpha$. The value of $\rho$ is in the interval $[0,1]$. To test the significance of the correlation Bartlett's criterion, Roy's criterion [42], bootstrap method [43] can be employed. In most of the applications the covariance matrices are singular, as the effective ranks of matrix are much lower than their sizes due to the small sample size and high-dimensional case. A common approach to deal with singular covariance matrices and to control complexity is ridge-type regularization. Here, we use 
an approach which maps the high-dimensional feature space onto a subspace with lower dimension, under the premise of not losing any valid information from the original samples. We assume that $\Sigma_{11}$ is singular and $\Sigma_{22}$ is nonsingular, and $v_{1}, v_{2}, \ldots, v_{m}$ (where $m=\operatorname{rank}\left(\Sigma_{11}\right)$ ) are its standard orthogonal eigenvectors corresponding to the nonvanishing eigenvalue of $\Sigma_{11}$, let $P=\left(v_{1}, v_{2}, \ldots, v_{m}\right)$, and replace $\Sigma_{11}$ and $\Sigma_{12}$ by $\tilde{\Sigma}_{11}=P^{T} \Sigma_{11} P$ and $\tilde{\Sigma}_{12}=P^{T} \Sigma_{12}$, respectively [44]. A similar approach is employed when both $\Sigma_{11}$ and $\Sigma_{22}$ are singular. It has been proven that the value of canonical correlation does not change when this dimension-reduction algorithm is applied [44].

In summary, the vector $Y$ is said to cause $X$ in linear Granger if, for some $t$, canoncorr $\left(\tilde{X}_{t}, Y_{t-\text { past }} \mid X_{t \text {-past }}\right) \neq 0$, that is, if the canonical correlation between $\tilde{X}_{t}$ and $Y_{t-\text { past }}$, partialing out the effect of $X_{t-p a s t}$ is different from zero. Here $\tilde{X}_{t}$ denotes $X_{t}$ once that the instantaneous effect of $Y_{t}$ has been partialed out. We consider $C C A(Y \rightarrow X)$ as the strength of causal influence from $Y$ to $X$, and we define causality from $X$ to $Y$ in a symmetric fashion.

We now address instantaneous causality by CCA. According to the previous definition of instantaneous causality [2], [11], the vector $X$ is said to instantaneously cause $Y$ in Granger sense if, for some $t$, canoncorr $\left(X_{t}, Y_{t} \mid\left[X_{t-\text { past }}, Y_{t-\text { past }}\right]\right) \neq 0$, that is, if canonical correlation between $X_{t}$ and $Y_{t}$, partialing out the effect of $X_{t-\text { past }}$ and $Y_{t-\text { past }}$, is nonzero.

\section{B. CCA Multivariate Granger Causality Analysis}

When applied to multivariate systems, bivariate Granger causality may detect spurious causalities due to the influence of the remaining time series [7], [12]. Multivariate Granger causality (MGC) was originally introduced by Geweke to solve the problem of indirect causal interaction [7], [45], [46], that is, causal interaction between two time series possibly influenced by other time series. Several other algorithms to detect multivariate causality in frequency domain have been developed, as (generalized) partial directed coherence and directed transfer function; both of them have been extended to exclude instantaneous effects [25], [26]. In the following, an extension of CCA bivariate Granger causality to the multivariate case in time domain is proposed. Let $Z(t)=\left[z_{1}(t), z_{2}(t), \ldots, z_{k}(t)\right]$ be $k$ simultaneously recorded multiple time series, which can be modeled by a traditional multivariate VAR process as following:

$$
Z(t)=\sum_{i=1}^{\infty} A_{i 0} Z_{t-i}+E_{0}(t) .
$$

Similarly, we consider the model which includes the instantaneous effects:

$$
Z(t)=\sum_{i=0}^{\infty} A_{i} Z_{t-i}+E(t) .
$$

Similarly to what we have demonstrated above, the vector $z_{i}$ is said to cause $z_{j}$ in linear Granger sense on information set $Z$ if, for some $t$, canoncorr $\left(\left\{\tilde{z}_{j}\right\}_{t},\left\{z_{i}\right\}_{t-\text { past }} \mid\left[Z_{t-\text { past }} \backslash\left\{z_{i}\right\}_{t-\text { past }}\right]\right) \neq 0$ (canonical correlation between $\left\{\tilde{z}_{j}\right\}_{t}$ and $\left\{z_{i}\right\}_{t-p a s t}$, partialing out the ef- fect of $Z_{t \text {-past }} \backslash\left\{z_{i}\right\}_{t \text {-past }}$ is nonzero, where $\left\{\tilde{z}_{j}\right\}_{t}$ denotes $\left\{z_{j}\right\}_{t}$ partialing out the instantaneous effect of $Z_{t} \backslash\left\{z_{j}\right\}_{t}$, $Z_{t-\text { past }} \backslash\left\{z_{i}\right\}_{t-\text { past }}$ denotes the information coming from data other than the values in $\left.\left\{z_{i}\right\}_{t-p a s t}\right)$. Repeating these steps for all the values of $i$ and $j$, the causality pattern in the dataset is evaluated.

In short, we give the pseudocode for calculating CCA multivariate Granger causality from multiple time series $z_{i} \in \Re^{N \times p}$ to $z_{j} \in \Re^{N \times q}$ (we denote $z_{a}=\left[z_{a, 1}, \ldots, z_{a, b}\right] \in$ $\Re^{N \times b}, \quad z_{a}^{m+1: N}=\left[z_{a, 1}^{m+1: N}, \ldots, z_{a, b}^{m+1: N}\right], \quad z_{a}^{1: N-m}=$ $\left[z_{a, 1}^{1: N-m}, \ldots, z_{a, b}^{1: N-m}\right]$, where $z_{a, h}=\left[z_{a, h}(1), \ldots, z_{a, h}(N)\right]^{T}$, $z_{a, h}^{m+1: N}=\left[z_{a, h}(m+1), \ldots, z_{a, h}(N)\right]^{T}, \quad z_{a, h}^{1: N-m}=\left[z_{a, h}(1)\right.$, $\left.\ldots, z_{a, h}(N-m)\right]^{T}$ in matrix form):

1) Preprocess the data and get multiple time series $Z \in \Re^{N \times n}$. Without loss of generality, let $Z=$ $\left[z_{i}, z_{j}, z_{3}, \ldots, z_{k}\right]$.

2) Select the lag order $m$ for $Z$ by some criterion.

3) Let $\quad z_{r}=\left[z_{3}, \ldots, z_{k}\right] \in \Re^{N \times(n-p-q)}, \quad z_{r i-c}=$ $\left[z_{r}^{m+1: N}, z_{i}^{m+1: N}\right], \quad z_{r j-p}=\left[z_{r}^{1: N-m}, z_{j}^{1: N-m}\right], \quad z_{j-c}=$ $z_{j}^{m+1: N}$ and $z_{i-p}=z_{i}^{1: N-m}$.

4) Remove influence of multiple lags. This could be achieved in two ways:

i) First, estimate conditional covariance matrix $\Sigma_{\tilde{z}_{j-c} \tilde{z}_{j-c}}=\Sigma_{z_{j-c} z_{j-c}} \mid z_{r i-c}$, then estimate conditional covariance matrix $\Sigma_{\tilde{z}_{j-c} z_{i-p} \mid z_{r j-p}}$.

ii) Regress out the influence of $z_{r i-c}$ on $z_{j-c}$, then get the residual vector $\tilde{z}_{j-c}$, partialing out the influence of $z_{r j-p}$ on $\tilde{z}_{j-c}$ and $z_{i-p}$, then get the residual vectors $\hat{z}_{j-c}$ and $\hat{z}_{i-p}$.

5) If you choose step 4.i), calculate the square root of maximum eigenvalue of matrix $A=R R^{T}$ (where $\left.R=\Sigma_{\tilde{z}_{j-c} \tilde{z}_{j-c} \mid z_{r j-p}}^{-1 / 2} \Sigma_{\tilde{z}_{j-c} z_{i-p} \mid z_{r j-p}} \Sigma_{z_{i-p} z_{i-p} \mid z_{r j-p}}^{-1 / 2}\right)$, which is $C C A\left(z_{i} \rightarrow z_{j}\right)$. If the covariance matrix is singular, we add a dimension reduction step following the strategy proposed in Section II (A).

For step 4.ii), calculate the canonical correlation between $\hat{z}_{j-c}$ and $\hat{z}_{i-p}$ (i.e., $C C A\left(z_{i} \rightarrow z_{j}\right)$ ) and the corresponding statistical significant level (for example using MATLAB function canoncorr).

\section{MATERIALS AND MEthodS}

\section{A. Simulation Model 1}

First, here we considered a AR(2) model:

$$
\begin{aligned}
& x(t)=0.95^{2} x(t-2)+0.4 y(t-2)+0.5 \varepsilon(t) \\
& y(t)=0.95^{2} y(t-2)+0.5 x(t)+c \xi(t)
\end{aligned}
$$

where $\varepsilon(t), \xi(t)$ are zero-mean uncorrelated Gaussian white noise processes with unit variances. We used various values of noise amplitude $c$ and various lengths $N$ of the time series to show how the two parameters affect the causality index. Then we repeated the simulation 200 times with random values of $\varepsilon$ and $\xi$ keeping $c$ and $N$ fixed in order to generate a null distribution (in order to ensure reliability of the result and calculate the mean 
and variance of the causal strength). We evaluated four types of GC with lag order 2: Geweke's GC $\left(F_{Y \rightarrow X}=\ln \frac{\left|\Sigma\left(\varepsilon_{t}\right)\right|}{\left|\Sigma\left(\varepsilon_{t}^{\prime}\right)\right|}[45]\right)$, improved Geweke's GC $\left(F_{Y \rightarrow X}=\ln \frac{\left|\Sigma\left(\varepsilon_{t}\right)\right|}{\left|\Sigma\left(\varepsilon_{t}^{\prime \prime}\right)\right|}\right.$, based on (1) and (3), CCA Standard GC (without excluding the instantaneous effect, i.e., $\mathrm{CCA}(Y \rightarrow X)=\operatorname{canoncorr}\left(X_{t}, Y_{t \text {-past }} \mid X_{t \text {-past }}\right)$ [21]), CCA GC (which excludes the instantaneous effect, i.e., $\left.\operatorname{CCA}(Y \rightarrow X)=\operatorname{canoncorr}\left(\tilde{X}_{t}, Y_{t-\text { past }} \mid X_{t-\text { past }}\right)\right)$.

\section{B. Simulation Model 2}

As a further test for the proposed approach, we considered a random vector model as following:

$$
\begin{aligned}
x_{1}(t)= & 0.8 x_{1}(t-1)-0.3 x_{1}(t-2)+0.5 x_{2}(t-1)+\varepsilon_{1}(t) \\
x_{2}(t)= & 0.4 x_{2}(t-2)+\frac{1.2-0.5 x_{1}(t-2)}{1+e^{-3 x_{1}(t-2)}}+\varepsilon_{2}(t) \\
y_{1}(t)= & -0.5 y_{1}(t-1)+0.75 z(t-2)+\varepsilon_{3}(t) \\
y_{2}(t)= & 0.5 y_{1}(t-2)+0.7 y_{2}(t-3)+\varepsilon_{4}(t) \\
z(t)= & 0.75 x_{1}(t-2)-0.5 x_{2}(t-2)+0.25 \sqrt{2} z(t-2) \\
& +\varepsilon_{5}(t)
\end{aligned}
$$

where $\varepsilon$ 's are unit variance Gaussian noise as above. Using the model simulation we generated a dataset of 500 time points. Then we evaluated the element-wise conditional causality for all pairs of maps. The lag order $(=3)$ was determined by leave-one-out cross-validation (LOO-CV). We define block sets as following combination: $X=\left\{x_{1}, x_{2}\right\}, Y=\left\{y_{1}, y_{2}\right\}, x z=$ $\left\{x_{1}, x_{2}, z\right\}, y z=\left\{y_{1}, y_{2}, z\right\}$. From the many ways in which $x_{1}, x_{2}, y_{1}, y_{2}$, and $z$ can be combined, we have chosen some typical blockwise architectures. In (8), the block-to-block causal relationships: $X \rightarrow Y, Y \rightarrow X, x z \rightarrow Y, Y \rightarrow x z, X \rightarrow y z$, and $y z \rightarrow X$, and the block to individual or individual to block causal interaction: $X \rightarrow z, z \rightarrow X, z \rightarrow Y, Y \rightarrow z, x_{1} \rightarrow y z$, $y z \rightarrow x_{1}, x_{2} \rightarrow y z, y z \rightarrow x_{2}$ were evaluated by CCA bivariate and multivariate Granger causality test. We repeated the simulation 200 times with a random $\varepsilon$ to generate a null distribution.

\section{Application to Scalp-EEG and Depth-EEG in Epilepsy}

Mesial temporal lobe epilepsy (mTLE) is the most common type of human medically intractable epilepsy. Surgical removal of brain tissue involved in seizure generation (epileptogenic zone, EZ) is at the moment the most effective treatment [47]. Correct identification of the epileptic focus that will be removed represents a crucial step for a successful outcome. To this purpose, both scalp-EEG and depth-EEG are recorded in the presurgical phase to investigate spatiotemporal dynamics of ictal and interictal states. Alongside the individuation of the focus and the study of how seizures initiate, propagate, and terminate, studying the dynamic relationships between different brain regions plays an important role. Several studies have analyzed EEG during seizures with different methods in order to localize the focus [48]-[50]. In this study, we concentrate on the interictal phase [51]-[53], looking for signs of seizure susceptibility and increased unilateral influence between hemispheres. Indeed, findings from brain-imaging studies in epilepsy have shown fea-

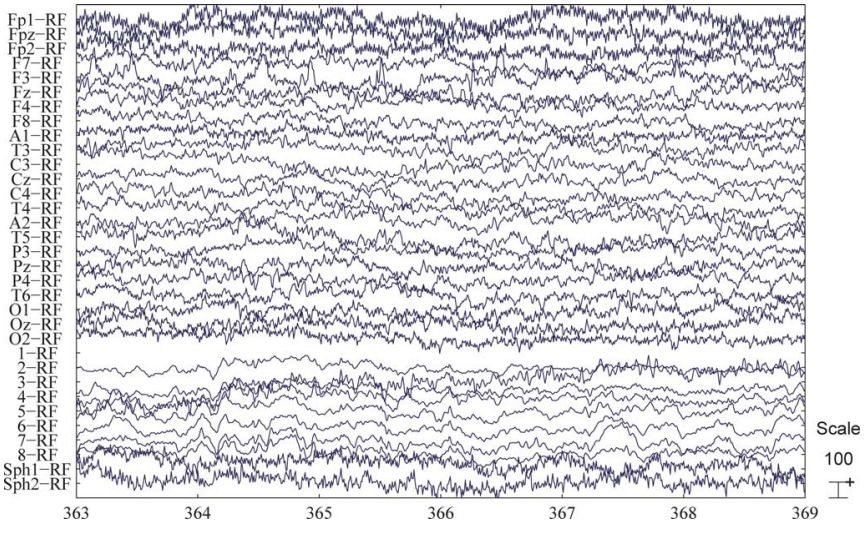

Fig. 1. Selection of interictal data. Plot obtained using EEGLAB.

tures which are not related to specific focal region dysfunction but to wider network dysfunctions [54]. Furthermore, the study of network dynamics can help to evaluate possible consequences of the surgery on subsequent brain functionality, once that the patient will be hopefully seizure-free.

We applied the proposed approach to test connectivity in simultaneously recorded scalp-EEG and depth-EEG data from one patient (female, 20 years) with medically refractory mesial temporal lobe epilepsy. Written Informed Consent was obtained from the participant. This study was approved by the local medical ethics committee at the First Affiliated Hospital, Fujian Medical University. The patient subsequently underwent surgical resection of the putative epileptogenic zone, is now seizure free, one year after surgery. The epileptogenic foci had been localized and evaluated by:

1) Structural MRI evidence of reduced volume of the right hippocampus relatively to the average normal Chinese hippocampus volume measured in coronal T1 images.

2) Increase in T2 fluid-attenuated inverted recovery signal in bilateral hippocampus, indicating that here was no other MRI abnormality than the hippocampal sclerosis.

3) EEG evidence: The patient showed spike discharges on right frontal lobe and bilateral sphenoidal electrodes during interictal period. The slow waves originated from the left sphenoidal electrode and propagated to the right sphenoidal electrode; frontal and temporal lobes showed spike discharges during ictal period.

Intracortical depth electrodes (interelectrode distance: $10 \mathrm{~mm}$ ) were placed on left and right hippocampal head, body, and posterior. For this patient, an anterior temporal lobectomy was performed. The left anterior temporal lobe, $4.5 \mathrm{~cm}$ from the temporal pole, the left amygdala, the hippocampal body and head, and the parahhippocampal gyrus were resected. Scalp EEG and depth-EEG were obtained at a sampling rate of $128 \mathrm{~Hz}$. Here we chose an interictal scalp-EEG and depth-EEG data for further analysis; the data are displayed in Fig. 1.For the scalp-EEG data, we only considered the Gamma rhythm wave (30 HZ high-pass filter) [47], which is stationary. First, we performed a CCA multivariate Granger causality analysis between each possible pairs of Gamma rhythm scalp-EEG signals [the lag is 8 determined by LOO-CV (Fig. 2)]. Finally, 

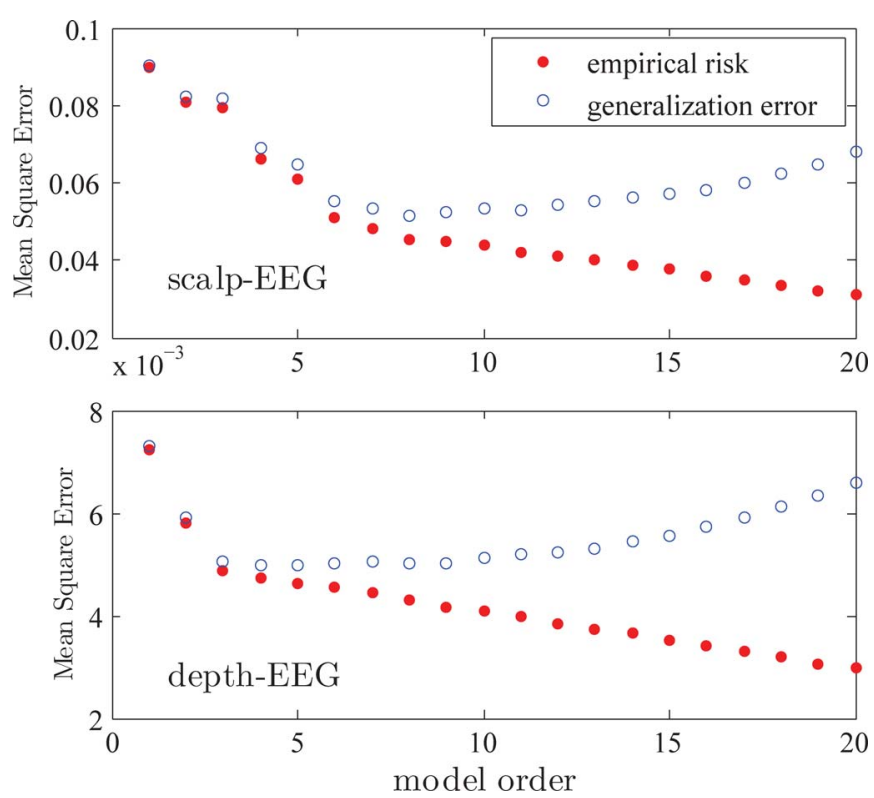

Fig. 2. LOO-CV plots for EEG data.

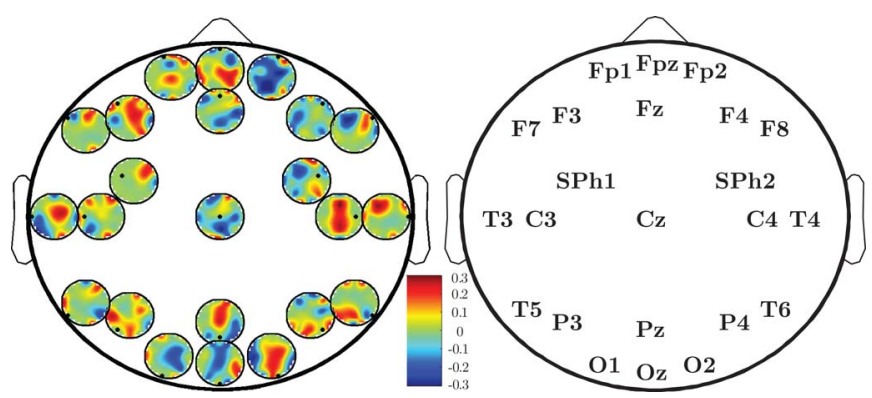

Fig. 3. (Left) Gamma rhythm causal connectivity map obtained by means of CCA multivariate Granger causality. Here, each small circle shows the causal flow (difference between outgoing and ingoing causalities) from each channel to all other channels. Positive values (red) indicate sources, and negative values (blue) indicate sinks of information. All the nonzero values have passed the significance test with a threshold of $q=0.05$, FDR corrected. (Right) The corresponding topographic map of the electrodes.

we analyzed the time-invariant and dynamic (we use moving windows with $1.25 \mathrm{~s}$ length) CCA bivariate Granger causality between two modules of the depth-EEG data [first-order differences were performed, in order to get a stationary data, the lag is 4 for time-invariant (Fig. 2) and 2 for dynamic case, which were determined by LOO-CV]. The LOO-CV plots for EEG data with time-invariant case are presented in Fig. 2, and the analysis results are presented in Fig. 3 .

\section{RESUlts AND DisCUSSION}

\section{A. Simulation Model 1}

For simulation model 1 , the causal interactions between $x$ and $y$ are displayed in Figs. 4 and 5 for different noise levels $c \in[0.1,1.5]$, different length of time series and lag distributions, respectively. The nonzero values are those that pass the significance test at a threshold of 0.05 , corrected by FDR [55].
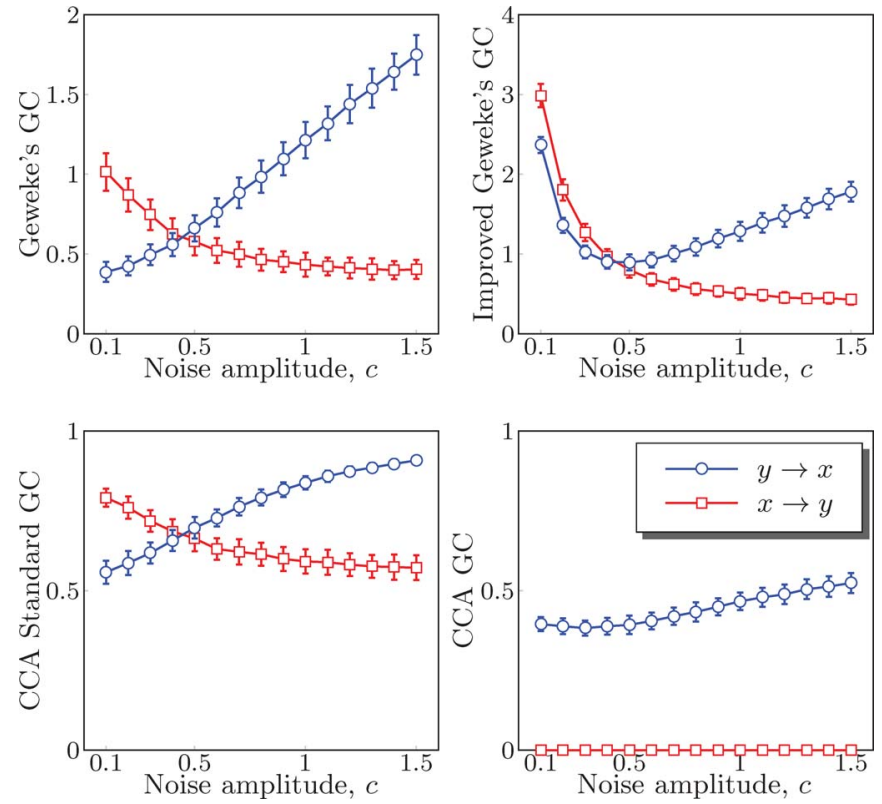

Fig. 4. Four types of Granger causal influences between simulated time series for increasing the noise levels. Left: Geweke's GC (top) and CCA Standard GC (bottom). Right: Improved Geweke's GC (top) and CCA GC (bottom). All nonzero values have passed the significance test at a confidence level of 0.05 , corrected by FDR.

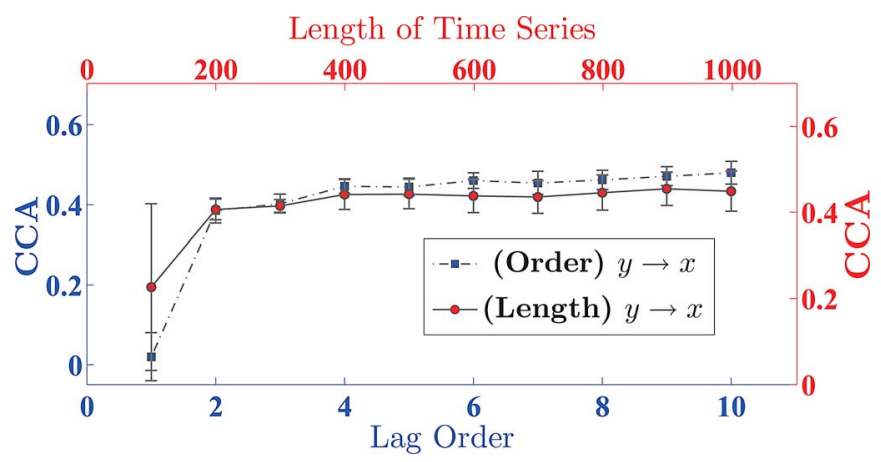

Fig. 5. Strength of causal influence affected by two factors: The length of the considered time series and the order of lag distribution.

Fig. 4 shows that traditional GC suffers from instantaneous causality, and even the improved Geweke's GC sometimes is unable to avoid it, while the proposed approach eliminates this problem. it is evident from Fig. 5 that Granger causal influence $y \rightarrow x$ significantly increases when we change the model order from 1 to 2, and then it stays more stable for bigger model orders, which indicates the risk caused by spurious regressions. It shows that the analysis results of Granger causality are stable when there are sufficient amount of realizations.

\section{B. Simulation Model 2}

For simulation model 2, the schematic connectivity plot for the simulation is displayed in Fig. 6. The results of bivariate and multivariate Granger causality analysis are displayed in the Figs. 7 and 8, respectively. Comparing the network diagrams for the two cases we can see that bivariate analysis yields connections that are the result of direct causal influences 


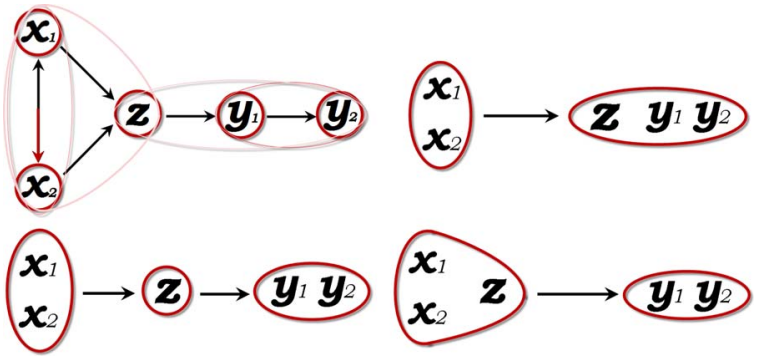

Fig. 6. Possible connectivities for the random vector model. Top left: The selective causal relationships between the variables in (8). The remaining diagrams show the patterns obtained by decomposing them.

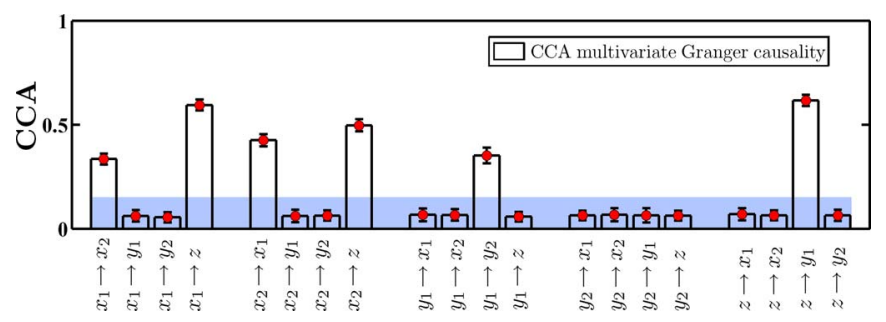

Fig. 7. CCA multivariate Granger causality values for all pairs of maps in (8). The light blue surface contains the nonsignificant values.

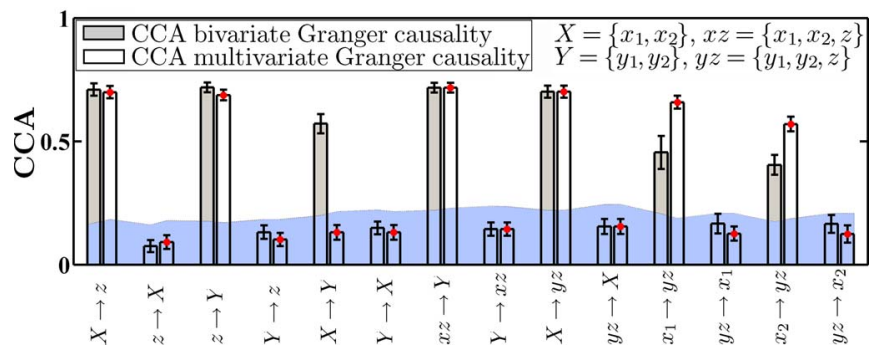

Fig. 8. CCA bivariate and multivariate Granger causality values among combinations of the variables of (8). The light blue surface contains the nonsignificant values.

(e.g., $X \rightarrow z$ ) and indirect causal influences (e.g., $X \rightarrow Y$ ) . We further performed a CCA multivariate Granger causality analysis. The results demonstrate that CCA multivariate Granger causality better matches the original structural connectivity. It is also worth noting that causal influence $x_{1} \rightarrow x_{2}$ is nonlinear, but it still can be detected by a linear test. When we group the variables, we observe that the group effect is different from the individual effect: for instance, the causal influence $X \rightarrow y z$ is stronger than the causal effect of $x_{1}$ and $x_{2}$ on $y z$ taken separately. This is in line with the redundancy effect described in [56].

\section{Scalp-EEG and Depth-EEG Data}

For the scalp-EEG (Fig. 3), it is shown that there is information flow transfer between two hemispheres, notably a higher information transfer from left to right hemisphere in the causality connectivity map [in particular, there is a significant causal influence from left sphenoidal electrode (SPh1) to the right one (SPh2)]. The time-invariant and dynamic causal analysis result
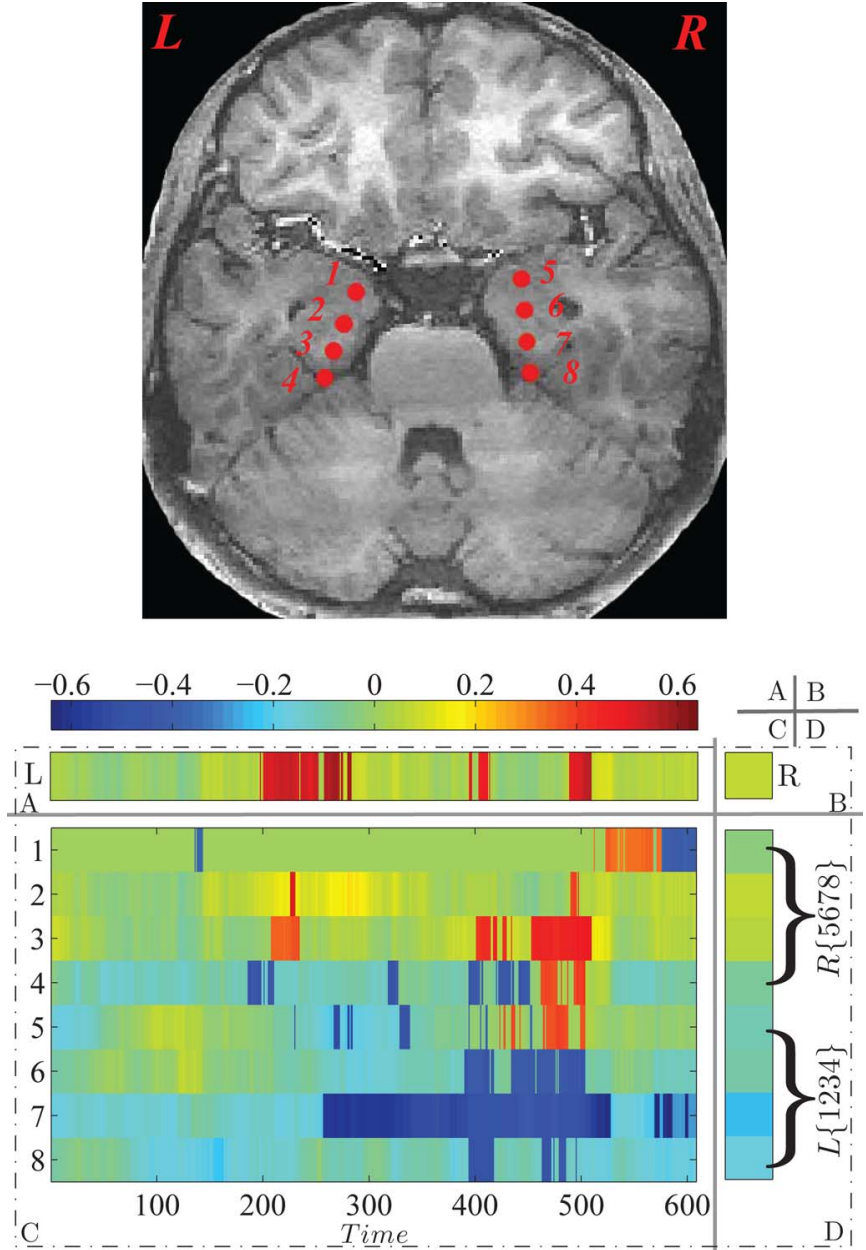

Fig. 9. Causal connectivity between modules. (Top) The intracranial electrode position (red solid spheres) is reconstructed by fusion technique of CT and MRI brain images. (Bottom) Information flow (outgoing minus ingoing causal relationships, i.e., those who have passed the significance test with $q=0.05$, FDR corrected) for time-invariant and dynamic CCA bivariate GC analysis. A: Dynamic information flow from left block electrodes to right block electrodes. B Time-invariant information flow from left block electrodes to right block electrodes. C: Dynamic information flow from single electrode to block electrodes. D: Time-invariant information flow from single electrode to block electrodes. E: The last row (all negative values) in $\mathrm{C}$ indicates that electrode 8 receives information from left block electrodes.

of simultaneously recorded depth-EEG confirms the analysis result of scalp EEG (Fig. 9). We find that the causal influence from the left module of four electrodes to the right module of four electrodes is more elevated than the one in the opposite direction, in particular electrodes 2 and 3 act as sources of information to right block electrodes, and electrodes 7 and 8 act as sinks of information coming from left block electrodes. This evidence of a preferred coupling direction, both from scalp and depth EEG, could add useful information on the epileptogenic zone, even in absence of seizures, as pointed out in [53]. Furthermore, this methodology provides a map of the brain connectivity in the interictal phase, which could possibly be useful to make predictions on the network disruption and reorganization after surgery [57], [58]. 


\section{Statistical Inference}

Several approaches are used to test the significance of VARbased Granger causality: F-test, $\chi^{2}$-test [2], [45], and the bootstrap method [6], [43]. In the present study, as the algorithm for Granger causality analysis is based on the canonical correlation, many validated methods to test the statistical significance of canonical correlation could also be employed. To test for statistical significance of canonical correlation coefficients, several asymptotic tests could be used, such as Fapproximations of Wilks' Lambda, the Hotelling-Lawley Trace, the Pillai-Bartlett Trace, or of Roy's Largest Root [59], and a Monte Carlo method [43]. Here, we use the Lawley's modification to Bartlett's chi-squared statistic.

\section{E. Influence of Order, Length and Dimension of Time Series on Granger Causality}

VAR-based Granger causality analysis must balance model order and complexity, and the corresponding estimation of AR model coefficients [2]. The lag order of the model is the main issue in the proposed approach, but we avoid potential parameter estimation problems that often arise multicollinearity [19], [20]. Furthermore, small sample size and high dimensionality are a common problem in real data (e.g., fMRI), which adds to the problem of lag order. To deal with this problem, we map the high-dimensional feature space onto a reduced dimensional subspace, without losing any valid information on the original samples. Nonetheless, the lag order and the number of multivariate time series (when combining the element for blockwise analysis) should always be chosen with caution. In this paper, we used cross validation based on Leave-One-Out error [34], [35], which uses all but a single data point of the original sample for training the model, and is defined as the averaged error:

$$
\operatorname{err}_{L O O}=\frac{1}{n} \sum_{i=1}^{n} l\left(y_{i}, f\left(x_{i} \mid D \backslash D_{i}\right)\right)
$$

where $D=\left\{\left(x_{1}, y_{1}\right), \ldots,\left(x_{n}, y_{n}\right)\right\}$ represents the original sample set of $n$ labeled, $D_{i}=\left(x_{i}, y_{i}\right), f\left(x_{i} \mid D \backslash D_{i}\right)$ denotes the model prediction at the instance $x_{i}$, and $l$ is $0 / 1$-loss function $(l(a, b)=0$, when $a=b$, otherwise, $l(a, b)=1)$.

\section{F. Blockwise Causal Interaction}

Detection of elementwise causal interaction is a popular method in the study of complex systems. Recently, more and more attention has been devoted to explore multidimensional signals using blockwise causal interactions [21], [45], [60], [61], [62]. In the interaction between several elements of a system we could have synergy, redundancy, and independence [56]. There are various ways to combine series into communities, using for example information from the geometric location, or some artificial designation. On the other hand, blockwise causal analysis may be used first to identify important system interactions, and then elementwise causal analysis can be used to further assess the contributions of specific subsystem within and between those systems [62]. The analysis of interactions at different scales brings a new insight on the interaction among complex subsystems.

\section{G. Limitations}

Linear Granger causality tests are useful to uncover linear and, in some particular case, even nonlinear causal relationships. However, their power in revealing nonlinear causal relationships can be low in many cases. Indeed, even if the dynamics of each individual element is nonlinear, it is still difficult to determine whether the corresponding collective dynamic could result in nonlinear relationships. Granger causality analysis can be performed in the time-domain as well as in the frequency-domain. The proposed approach is purely a time domain test; if one is interested to frequency domain results in this framework, a possible solution is to preprocess the data, for example filtering them (although this might bring to some other issues [63]). The framework of Granger causality assumes stationarity of signals, thus Granger causality estimates are prone to lose validity when signals violate stationarity assumptions. Furthermore, the pitfalls of this approach considering the inevitable occurrence of trial-to-trial variability of event-related potentials in both amplitudes and latencies have been pointed out [64].

\section{CONCLUSION}

CCA is a popular multivariate statistical method which is extensively used to analyze time series data. In this work, we proposed a novel canonical correlation algorithm for multivariate Granger causality analysis, which greatly decreases computation complexity compared to parametric estimators. In order to overcome common problems of singular in the covariance matrix, reducing the dimension of covariance matrix is added to the algorithm which makes the test more robust. We believe that the proposed approach is a simple and yet powerful statistical method able to assess causal relationships in multivariate systems and to provide a deeper understanding of the underlying information flow at several scales in complex networks in general and brain networks in particular. Importantly, its application could shed light on clinically relevant phenomena such as epilepsy, as shown here.

\section{ACKNOWLEDGMENT}

The authors thank Wei Liao and Zhiqiang Zhang, Department of Medical Imaging, Jinling Hospital, Nanjing, China, and anonymous reviewers for their useful comments on this study.

\section{REFERENCES}

[1] P. A. Valdes-Sosa, A. Roebroeck, J. Daunizeau, and K. Friston, "Effective connectivity: Influence, causality, and biophysical modeling," NeuroImage, vol. 58, pp. 339-361, 2011.

[2] S. L. Bressler and A. K. Seth, "Wiener-Granger causality: A well established methodology," Neuroimage, vol. 58, pp. 323-329, 2011.

[3] D. Marinazzo, W. Liao, H. Chen, and S. Stramaglia, "Nonlinear connectivity by Granger causality," NeuroImage, vol. 58, pp. 330-338, 2011.

[4] C. Buchel and K. J. Friston, "Modulation of connectivity in visual pathways by attention: Cortical interactions evaluated with structural equation modelling and fMRI," Cerebral Cortex, vol. 7, no. 8, p. 768, 1997.

[5] K. J. Friston, L. Harrison, and W. Penny, "Dynamic causal modelling," Neuroimage, vol. 19, no. 4, pp. 1273-1302, 2003. 
[6] G. Deshpande, S. LaConte, G. A. James, S. Peltier, and X. Hu, "Multivariate Granger causality analysis of fMRI data," Human Brain Mapp., vol. 30, no. 4, pp. 1361-1373, 2009.

[7] M. Ding, Y. Chen, and S. L. Bressler, "Granger causality: Basic theory and application to neuroscience," in Handbook of Time Series Analysis, B. Schelter, et al. eds. New York: Willey-Vch, 2006, 2011.

[8] R. Goebel, A. Roebroeck, D. S. Kim, and E. Formisano, "Investigating directed cortical interactions in time-resolved fMRI data using vector autoregressive modeling and Granger causality mapping," Magn. Reson. Imag., vol. 21, no. 10, pp. 1251-1261, 2003.

[9] W. Liao, D. Marinazzo, Z. Pan, Q. Gong, and H. Chen, "Kernel Granger causality mapping effective connectivity on fMRI data," IEEE Trans. Med. Imag., , vol. 28, no. 11, pp. 1825-1835, 2009.

[10] W. Liao, J. Ding, D. Marinazzo, Q. Xu, Z. Wang, C. Yuan, Z. Zhang, G. Lu, and H. Chen, "Small-world directed networks in the human brain: Multivariate Granger causality analysis of resting-state fmri," NeuroImage, vol. 54, no. 4, pp. 2683-2694, 2011.

[11] K. Friston, "Causal modelling and brain connectivity in functional magnetic resonance imaging," PLoS Biol, vol. 7, no. 2, p. e1000033, 2009.

[12] C. W. J. Granger, "Economic processes involving feedback," Inf. Control, vol. 6, no. 1, pp. 28-48, 1963.

[13] C. W. J. Granger, "Investigating causal relations by econometric models and cross-spectral methods," Econometrica: J. Econometric Soc., vol. 37, no. 3, pp. 424-438, 1969.

[14] K. Hlavácková-Schindler, M. Palus, M. Vejmelka, and J. Bhattacharya, "Causality detection based on information-theoretic approaches in time series analysis," Phys. Reports, vol. 441, no. 1, pp. 1-46, 2007.

[15] A. J. Cadotte, T. B. DeMarse, T. H. Mareci, M. B. Parekh, S. S. Talathi, D. U. Hwang, W. L. Ditto, M. Ding, and P. R. Carney, "Granger causality relationships between local field potentials in an animal model of temporal lobe epilepsy," J. Neurosci. Methods, vol. 189, pp. 121-129, 2010.

[16] J. R. Sato, P. A. Morettin, P. R. Arantes, and E. Amaro Jr., "Wavelet based time-varying vector autoregressive modelling," Comput. Statist. Data Anal., vol. 51, no. 12, pp. 5847-5866, 2007.

[17] A. Fujita, J. R. Sato, K. Kojima, L. R. Gomes, M. Nagasaki, M. C. Sogayar, and S. Miyano, "Identification of Granger causality between gene sets," J. Bioinformat. Comput. Biol., vol. 8, no. 4, pp. 679-701, 2010.

[18] D. Marinazzo, M. Pellicoro, and S. Stramaglia, "Kernel method for nonlinear Granger causality," Phys. Rev. Lett., vol. 100, no. 14, p. 144103, 2008.

[19] P. W. Otter, "Canonical correlation in multivariate time series analysis with an application to one-year-ahead and multiyear-ahead macroeconomic forecasting," J. Business Econ. Statist., vol. 8, no. 4, pp. 453-457, 1990.

[20] P. W. Otter, "On Wiener-Granger causality, information and canonical correlation," Econ. Lett., vol. 35, no. 2, pp. 187-191, 1991.

[21] J. R. Sato, A. Fujita, E. F. Cardoso, C. E. Thomaz, M. J. Brammer, and E. Amaro Jr., "Analyzing the connectivity between regions of interest: An approach based on cluster Granger causality for fMRI data analysis," Neurolmage, vol. 52, pp. 1444-1455, 2010.

[22] D. Marinazzo, M. Pellicoro, and S. Stramaglia, "Kernel-Granger causality and the analysis of dynamical networks," Phys. Rev. E, vol. 77, no. 5, p. 56215, 2008.

[23] G. Wu, X. Duan, W. Liao, Q. Gao, and H. Chen, "Kernel canonicalcorrelation granger causality for multiple time series," Phys. Rev. E, vol. 83, no. 4 , p. $041921,2011$.

[24] L. A. Baccala and K. Sameshima, "Partial directed coherence: a new concept in neural structure determination," Biol. Cybern., vol. 84, no. 6, pp. 463-474, 2001.

[25] G. Deshpande, K. Sathian, and X. Hu, "Assessing and compensating for zero-lag correlation effects in time-lagged Granger causality analysis of fMRI," Biomed. Eng., IEEE Trans., vol. 57, no. 6, pp. 1446-1456, 2010.

[26] L. Faes and G. Nollo, "Extended causal modeling to assess Partial directed coherence in multiple time series with significant instantaneous interactions," Biol. Cybern., pp. 1-14, 2010.

[27] D. A. Pierce and L. D. Haugh, "Causality in temporal systems: Characterization and a survey," J. Econometr., vol. 5, no. 3, pp. 265-293, 1977.

[28] R. Ashley, C. W. J. Granger, and R. Schmalensee, "Advertising and aggregate consumption: an analysis of causality," Econometrica: J. Econometric Soc., vol. 48, no. 5, pp. 1149-1167, 1980.

[29] H. Lütkepohl, New Introduction to Multiple Time Series Analysis. New York: Springer-Verlag, 2005.

[30] A. B. Barrett, L. Barnett, and A. K. Seth, "Multivariate Granger causality and generalized variance," Phys. Rev. E, vol. 81, no. 4, p. 41907, 2010.

[31] H. Akaike, "A new look at the statistical model identification," IEEE Trans. Automat. Control,, vol. 19, no. 6, pp. 716-723, 2002.
[32] G. Schwarz, "Estimating the dimension of a model," Ann. Statist., vol. 6, no. 2, pp. 461-464, 1978.

[33] E. J. Hannan and B. G. Quinn, "The determination of the order of an autoregression," J. R. Statist. Soc. Series B (Methodological), vol. 41, no. 2, pp. 190-195, 1979.

[34] S. Lemm, B. Blankertz, T. Dickhaus, and K. R. Muller, "Introduction to machine learning for brain imaging," NeuroImage, vol. 56, no. 2, pp. 387-399, 2011.

[35] B. Efron and R. Tibshirani, "Improvements on cross-validation: The. 632+ bootstrap method," J. Amer. Statist. Assoc., vol. 92, no. 438, pp. 548-560, 1997.

[36] J. F. Geweke, "Measurement of linear dependence and feedback between multiple time series," J. Amer. Statist. Assoc., vol. 77, no. 378, pp. 304313, 1982.

[37] R. Rajagovindan and M. Ding, "Decomposing neural synchrony: toward an explanation for near-zero phase-lag in cortical oscillatory networks," PLoS One, vol. 3, no. 11, p. 3649, 2008.

[38] H. Hotelling, "Relations between two sets of variates," Biometrika, vol. 28, no. 3-4, p. 321, 1936.

[39] J. R. Kettenring, "Canonical analysis of several sets of variables," Biometrika, vol. 58, no. 3, p. 433, 1971.

[40] K. E. Muller, "Understanding canonical correlation through the general linear model and principal components," Amer. Statistician, vol. 36, no. 4 pp. 342-354, 1982.

[41] C. W. J. Granger and P. Newbold, "Spurious regressions in economics," J. Econometr., vol. 2, no. 2, pp. 111-120, 1974.

[42] N. H. Timm and J. E. Carlson, "Part and bipartial canonical correlation analysis," Psychometrika, vol. 41, no. 2, pp. 159-176, 1976.

[43] B. Efron, R. Tibshirani, and R. J. Tibshirani, An Introduction to the Bootstrap. London, U. K.: Chapman \& Hall/CRC, 1993.

[44] Q. S. Sun, S. G. Zeng, Y. Liu, P. A. Heng, and D. S. Xia, "A new method of feature fusion and its application in image recognition," Pattern Recognit., vol. 38, no. 12 , pp. 2437-2448, 2005.

[45] J. F. Geweke, "Measures of conditional linear dependence and feedback between time series," J. Amer. Statist. Assoc., vol. 79, no. 388, pp. 907915, 1984.

[46] W. Liao, D. Mantini, Z. Zhang, Z. Pan, J. Ding, Q. Gong, Y. Yang, and H. Chen, "Evaluating the effective connectivity of resting state networks using conditional Granger causality," Biol. Cybern., vol. 102, no. 1, pp. 57-69, 2010

[47] B. Schelter, Seizure Prediction in Epilepsy: From Basic Mechanisms to Clinical Applications. New York: VCH Pub., 2008.

[48] C. Wilke, W. Van Drongelen, M. Kohrman, and B. He, "Neocortical seizure foci localization by means of a directed transfer function method," Epilepsia, vol. 51, no. 4, pp. 564-572, 2010.

[49] F. H. Lin, K. Hara, V. Solo, M. Vangel, J. W. Belliveau, S. M. Stufflebeam, and M. S. Hamalainen, "Dynamic granger-geweke causality modeling with application to interictal spike propagation," Human Brain Mapp., vol. 30, no. 6, pp. 1877-1886, 2009.

[50] P. J. Franaszczuk and G. K. Bergey, "Application of the directed transfer function method to mesial and lateral onset temporal lobe seizures," Brain Topography, vol. 11, no. 1, pp. 13-21, 1998.

[51] K. Lehnertz, R. G. Andrzejak, J. Arnhold, T. Kreuz, F. Mormann, C. E. Rieke, G. Widman, and C. Elger, "Its possible use for interictal focus localization, seizure anticipation, and prevention: Nonlinear EEG analysis in epilepsy," J. Clin. Neurophysiol., vol. 18, no. 3, p. 209, 2001.

[52] A. Kraskov, T. Kreuz, R. Q. Quiroga, P. Grassberger, F. Mormann, K. Lehnertz, and C. E. Elger, "Comparison of two phase synchronization analysis techniques for interictal focus lateralization in mesial temporal lobe epilepsy," Epilepsia, vol. 43, no. 7, p. 48, 2002.

[53] A. V. Medvedev, A. M. Murro, and K. J. Meador, "Abnormal interictal gamma activity may manifest a seizure onset zone in temporal lobe epilepsy," Int. J. Neural Syst., vol. 21, no. 2, pp. 103-114, 2011.

[54] W. Liao, Z. Zhang, Z. Pan, D. Mantini, J. Ding, X. Duan, C. Luo, G. Lu, and H. Chen, "Altered functional connectivity and small-world in mesial temporal lobe epilepsy," PLoS One, vol. 5, no. 1, p. e8525, 2010.

[55] Y. Benjamini and Y. Hochberg, "Controlling the false discovery rate: a practical and powerful approach to multiple testing," J. R. Statist. Soc. Series B (Methodological), vol. 57, no. 1, pp. 289-300, 1995.

[56] D. Marinazzo, W. Liao, M. Pellicoro, and S. Stramaglia, "Grouping time series by pairwise measures of redundancy," in Phys. Lett. A, 2010.

[57] B. C. Bernhardt, Z. Chen, Y. He, A. C. Evans, and N. Bernasconi, "Graphtheoretical analysis reveals disrupted small-world organization of cortical thickness correlation networks in temporal lobe epilepsy," Cerebral Cortex, vol. 21, pp. 2147-2157, 2011. 
[58] J. Alstott, M. Breakspear, P. Hagmann, L. Cammoun, and O. Sporns, "Modeling the impact of lesions in the human brain," PLoS Comput. Biol., vol. 5, no. 6, p. e1000408, 2009.

[59] W. J. Krzanowski, Principles of Multivariate Analysis: A User'S Perspective. New York: Oxford Univ. Press, 2000.

[60] Y. Chen, S. L. Bressler, and M. Ding, "Frequency decomposition of conditional Granger causality and application to multivariate neural field potential data," J. Neurosci. Methods, vol. 150, no. 2, pp. 228-237, 2006.

[61] C. Ladroue, S. Guo, K. Kendrick, J. Feng, and V. Brezina, "Beyond element-wise interactions: Identifying complex interactions in biological processes," PLoS One, vol. 4, no. 9, p. e6899, 2009.

[62] X. Wang, Y. Chen, S. L. Bressler, and M. Ding, "Granger causality between multiple interdependent neurobiological time series: Blockwise versus pairwise methods," Int. J. Neural Syst., vol. 17, no. 2, p. 71, 2007.
[63] E. Florin, J. Gross, J. Pfeifer, G. R. Fink, and L. Timmermann, "The effect of filtering on Granger causality based multivariate causality measures," NeuroImage, vol. 50, no. 2, pp. 577-588, 2010.

[64] X. Wang, Y. Chen, and M. Ding, "Estimating Granger causality after stimulus onset: A cautionary note," NeuroImage, vol. 41, no. 3, pp. 767$776,2008$.

Author's photographs and biographies not available at the time of publication. 